P-414 MUSCULOSKELETAL DISORDERS OF THE UPPER LIMBS IN A JEANS WASH COMPANY: ABOUT 220 CASES

Nihel Khouja, Jihen Hsinet, Saloua Ismail, Ibtihel Riahi, 'Ines Aissa, Amani Dallagi, Emna Baraketi, Aida Benzarti, Abdelmajid Ben Jemaa. ${ }^{1}$ Faculty of Medicine of Tunis, Tunisia

\subsection{6/OEM-2021-EPI.318}

Introduction Musculoskeletal disorders (MSDs) of the upper limbs are the most common occupational pathology in Tunisia with an increasing prevalence in all sectors of activity, in particular in the jeans washing industry.

Objectives Identify the risk factors for MSDs in this industry and evaluate the impact of these disorders on professional life.

Methods We carried out a cross-sectional descriptive survey of employees in a jeans washing company. This study was based on a questionnaire inspired by two validated questionnaires, the Nordic and from the National Research and Safety Institute, allowing the screening of MSDs.

Results The sample consisted of 220 employees with an average age of 36 years, a clear predominance of men and an average professional length of service of 6.2 years. They mainly occupied the post of multi-skilled worker $(56.8 \%)$. These employees reported a high pace of professional tasks $(60.4 \%)$, significant muscular strength required (60.9\%) and recourse to extreme postures (66.3\%). Certain psychosocial factors were reported: precision work $(83.2 \%)$, requiring great concentration (87.7\%), described as monotonous (81.4\%) and not very motivating (64.1\%). Musculoskeletal disorders, evolving for $2.3 \pm 1.6$ months on average, were also reported by $71.8 \%$ of employees. They complained of pain in the shoulders (39.5\%), hands and wrists (11.8\%) and elbows $(2.7 \%)$. These MSDs justified medical treatment in $25.9 \%$ of cases and exploration by additional examinations in $18.6 \%$ of cases. These disorders had an impact on professional activity with a temporary stoppage of work $(6.8 \%)$ and professional reclassification (1.4\%) with a type of workstation arrangement or transfer to another workstation.

Conclusion The jeans wash sector is a source of MSDs of the upper limbs due to excessive exposure to biomechanical and psycho-organizational constraints. This justifies the implementation of an effective preventive strategy based on early detection and ergonomic study of workstations.

\section{P-422 MENTAL HEALTH AND LIFE SATISFACTION AMONG CANADIAN PARAMEDICS DURING THE COVID-19 PANDEMIC}

${ }^{1}$ Christopher MacDonald, Paul Demers, Brian Grunau, David Goldfarb, David O'Neill, Richard Armour, Tracy Kirkham. 'Ontario Health, Canada and University of Toronto, Canada

\subsection{6/OEM-2021-EPI.319}

Introduction Paramedics have treated many patients suspected of having COVID-19 throughout this pandemic. The uncertainty regarding COVID-19 contagion, effectiveness of infection protection and controls, and the uncontrolled environments in which paramedics work may adversely impact their mental health.

Objectives To characterize the impact of the COVID-19 pandemic on the mental health of Canadian paramedics using preliminary data.
Methods Self-reported questionnaire data was collected from paramedics across five Canadian provinces as part of the COVID-19 Occupational Risks, Seroprevalence and Immunity among Paramedics (CORSIP) project. Validated screening scores were calculated for major depressive disorder (MDD, PHQ-9 questionnaire) and probable post-traumatic stress disorder (PTSD, PC-PTSD-5 questionnaire). Satisfaction with life (SWL) measures were adapted from validated Canadian Census questions and confirmed by reliability analysis. Bivariate relationships of screening scores were analyzed using Wilcoxon signed-ranked, Cliff's d, and differences in proportions tests where appropriate.

Results Completed questionnaires from 1,906 recruited paramedics were analyzed ( $95 \%$ completion rate). The overall prevalence of MDD was $31.6 \%$. Suicidal ideation (i.e., 'thoughts that you would be better off dead, or of hurting yourself in some way') was reported by $8.3 \%$ of paramedics. In addition, $40.2 \%$ of paramedics screened positive for probable PTSD, with no significant difference being reported before vs. during the pandemic. Paramedics reported higher median SWL prior to the COVID-19 pandemic (20 vs. 16, $\mathrm{p}<0.001)$. There was a large effect size $(\mathrm{d}=0.52)$, suggesting a greater probability that paramedics would report higher SWL prior to COVID-19.

Conclusion Canadian paramedics appear to be at risk for MDD and PTSD. While the proportion of paramedics with probable PTSD does not appear to have changed during the pandemic, a reduction in SWL was observed. Incorporating follow-up data that will be collected from participants over the next year will be imperative to assess the stability of these findings and evaluate differences by province.

\section{P-428 LOCAL NARRATIVES ON VULNERABILITIES RELATED TO PESTICIDE EXPOSURE IN THE AGROCITY OF GUICHÓN, PAYSANDÚ, URUGUAY}

${ }^{1}$ Nicolás Rodríguez González, Andrés Trotta, Damián Herkovits. 'School of Psychology, Uruguay

\subsection{6/OEM-2021-EPI.320}

Introduction Pesticide exposure involves toxicological and epidemiological events, and the perception of risks as a socially constructed process. Despite the growing and widespread research of exposure among rural workers, very few studies have focused on the risk perception on other population groups, particularly in agrocity contexts. The lack of this kind of investigation in Uruguay has limited monitoring program development that includes social dimensions of pesticides exposure.

Objectives To describe and analyze discourses produced by educational, productive, health and social actors in the agrocity of Guichón, Uruguay, regarding health vulnerabilities caused by pesticides exposure.

Methods We selected Guichón-city as a paradigmatic case and conducted exploratory interviews among key actors in 2017. The selected population consisted of pesticide applicators, heads of local health services, school principals and members of non-governmental organizations. Sixteen semi-structured interviews were conducted between 2018 and 2020. In addition, regular visits were made to the city where the geographical environment and the relationship with pesticides were 
observed. Field material was processed through thematic content analysis.

Results We identified two discursive formations in tension in the delimited agrocity. One set of discourse was defined as productive-preventive. Among those discourse, pesticides vulnerabilities were individualised and relativised, agronomic sources were invoked, and preventive actions were privileged. On the other hand, environmental-health discourses refered to different diseases, loss of wildlife and biodiversity and community's concerns. Direct experience or exchange with peers support these discourses, which are articulated with practices of denounce.

Conclusions In the agrocity of Guichón, discourses about health vulnerabilities of pesticides are in dispute. On the one hand, there are defensive strategies that naturalize risks while on the other, risks are problematized and questioned. Promotion of dialogical networks appears to be an indispensable premise for the development of a local monitoring policy on this topic.

\section{P-429 BIOSAFETY IN THE FISH INDUSTRY: A CASE STUDY IN THE STATE OF RIO DE JANEIRO}

${ }^{1}$ Ana Luísa Reis Ribeiro, Ana Paula Chein Bueno de Azevedo, Telma Abdalla de Oliveira Cardoso. ${ }^{1}$ ESNP, Brazil and Fiocruz, Brazil

\subsection{6/OEM-2021-EPI.321}

Introduction The fish industry is a growing sector worldwide, due to the increase demand for its products. To make its products competitive and attractive to consumers, the industry uses strict quality standards.

Objective To identify and understand the environmental risks that workers in the fish processing industry are subjected to, based on the field of biosafety, health and safety at work.

Methods For this purpose, an observational study was carried out with the help of a script to survey the process and flows at work and the physical-environmental aspects in a company in Macaé, Rio de Janeiro. For the analysis of the collected data a documentary research was carried out, to survey the legal requirements, which contemplate aspects related to the field of biosafety, health and safety at work. In addition, a literature review was conduced to identify the main occupational diseases that can affect workers in the sector.

Results Workers were exposed to biological, chemical, physical, ergonomic and accident risks. Among the risks identify, we can highlight: intense physical effort, manual lifting and carrying of weight, inadequate posture, high pace, contact with biological samples, bioaerosols inhale, excessive noise, intense cold, excessive humidity, vibration, contact with chemical products, poor lighting, inadequate electrical installations, handling of sharp objects, and others. The literature review allowed us to identify the possibility of occupational asthma, work-related asthma, occupational rhinoconjunctivitis, musculoskeletal disorders (e.g. carpal tunnel syndrome, epicondylitis, and tendonitis), hives, and contact dermatitis.

Conclusion The recognition and evaluation of situations at risk in the fish processing industry is of great importance to avoid unnecessary exposure and to take measures to prevent, mitigate and control existing risks in order to preserve the health of the workers and other impacts on both society and the environment.

\section{P-430 ADVERSE CHILDHOOD EXPERIENCES AND WORKPLACE VIOLENCE VICTIMIZATION IN ADULTHOOD}

${ }^{1}$ Amira Omrane, Myriam Ouerchefani, Imen Mlouki, Taoufik Khalfallah, Lamia Bouzgarrou, Sana Mhamdi. ${ }^{1}$ Faculty of Medicine of Monastir, Tunisia

\subsection{6/OEM-2021-EPI.322}

Background Research concerning the long-term effects of child maltreatment and other adverse childhood experiences (ACEs) has increased over the past decades. These researches provided a strong connection between early experiences and optimal health, wellness, and life opportunities across the life course. Additionally, victims of ACEs are at increased risk for experiencing violence across their life with accumulating risk for poorer health and social outcomes. This study aims to evaluate associations of commonly co-occurring childhood adversities with workplace violence in healthcare sector.

Methods This was a cross-sectional bi-centric study conducted on healthcare workers of two Public hospitals in the centraleastern region of Tunisia for a period of eight months. A selfadministered questionnaire was used to assess the prevalence of workplace violence and its determinants. ACEs were evaluated by the Arabic version of the ACEs International Questionnaire (ACE-IQ) in its validated Arabic version.

Results A total of 546 healthcare workers completed the questionnaire. All respondents reported experiencing at least one ACE. Intra-familial ACEs were more frequent than social ACEs with 100\% $(n=546)$ and $80.4 \%(n=439)$ respectively. About $96 \%$ of respondents reported exposure to at least one violent incident throughout their entire career in public health settings. Non-physical violence $(95.8 \%)$ was more frequent thanphysical violence (26.4\%). Multiple logistic regression showed that workers with childhood experiences of peer violence and community violence were more likely to be exposed to physical violence ( $\mathrm{p}=0.005$ and 0.014 respectively).

Conclusion This study identified an alarming prevalence of workplace violence against healthcare workers in Tunisia. ACEs were also prevalent and identified as a determinant factor of adulthood victimisation in healthcare sector. Understanding the overlapping causes of violence and why some individuals are at greater risk for experiencing violence across their life is important, because it can help address and prevent violence.

\section{P-431 HEALTH AND COVID: THE WORK OF ENDEMIC WORKERS DURING THE COVID-19 PANDEMIC IN RIO DE JANEIRO, BRAZIL.}

${ }^{1}$ Marcus Santos, Gabriel Silveira, Ana-Paula Neves, Priscila Vidal, Victor Figueiredo, Luiza Dantas, Marcos-Rogerio Silva, Ébio-Willis Moreira, Roberto-Paulo Nunes, Socorro Setúbal, Antônio-Carlos Cardoso, Monica Martins, Fátima Moreira3, Edilene Pereira, Tatiana Docile, Aline Gurgel, Márcia Sarpa, Leandro Carvalho, Luciana Gomes, Maria-Blandina Santos, Luiz-Claúdio Meirelles, Ana-Cristina Rosa, Eline Gonçalves, Liliane Teixeira, Ariane Larentis, Isabele Costa-Amaral. ${ }^{1}$ Fiocruz, Brazil

\subsection{6/OEM-2021-EPI.323}

Introduction The Brazilian vectorial 'control' is characterized by the intensive use of pesticides (agrotoxics), such as organochlorines, organophosphates, carbamates, pyrethroids, benzoylureas; some of them banned worldwide or restricted by international agreements. Continuous exposure to these neurotoxic and/or carcinogenic products has resulted in damage to the health of Endemic Workers, leading to a process of illness and deaths. Thus, because they have chronic diseases and are 